\title{
Sotos syndrome and haploinsufficiency of NSD 1: clinical features of intragenic mutations and submicroscopic deletions
}

\author{
T Nagai, N Matsumoto, N Kurotaki, N Harada, N Niikawa, T Ogata, K Imaizumi, \\ K Kurosawa, T Kondoh, H Ohashi, M Tsukahara, Y Makita, T Sugimoto, T Sonoda, \\ T Yokoyama, K Uetake, S Sakazume, Y Fukushima, K Naritomi
}

S otos syndrome (MIM 117550) is a congenital developmental disorder characterised by overgrowth and advanced bone age in infancy to early childhood, mental retardation, and various minor anomalies such as macrocephaly, prominent forehead, hypertelorism, downward slanting palpebral fissures, large ears, high and narrow palate, and large hands and feet. ${ }^{12}$ It is also frequently associated with brain, cardiovascular, and urinary anomalies ${ }^{3-6}$ and is occasionally accompanied by malignant lesions such as Wilms tumour and hepatocarcinoma. ${ }^{7}$ This condition has been classified as an autosomal dominant disorder, because several familial cases consistent with dominant inheritance have been described previously. ${ }^{9}$ Thus, sporadic cases accounting for most of the Sotos syndrome patients are assumed to be the result of de novo dominant mutations.

We have recently shown that Sotos syndrome is caused by haploinsufficiency of the gene for NSDl (nuclear receptor binding Su-var, enhancer of zeste, and trithorax domain protein 1$) \cdot{ }^{10} \mathrm{NSD} l$ consists of 23 exons and encodes at least six functional domains possibly related to chromatin regulations (SET, PWWP-I, PWWP-II, PHD-I, PHD-II, and PHD-III), in addition to 10 putative nuclear localisation signals. ${ }^{11}$ It is expressed in several tissues including fetal/ adult brain, kidney, skeletal muscle, spleen, and thymus ${ }^{11}$ and is likely to interact with nuclear receptors as a bifunctional transcriptional cofactor. ${ }^{12}$ In this paper, we report on clinical findings in Japanese patients with proven point mutations in NSD1 and those with submicroscopic deletions involving the entire NSDl gene and discuss genotype-phenotype correlation.

\section{METHODS}

This study consisted of five patients with heterozygous NSDI point mutations and 21 patients with heterozygous submicroscopic deletions involving the entire NSDl gene. The mutations were identified by direct sequencing of exons 2-23 and their flanking introns covering the whole coding region of NSD $1,{ }^{11}$ using genomic DNA extracted from peripheral leucocytes or lymphoblastoid cell lines. They included a novel nonsense mutation in exon 7 (3958C > T, R1320X), in addition to the previously reported four mutations: a nonsense mutation in exon 5 (1310C $>$ G, S437X), a one base deletion in exon 5 (3356delA) resulting in a premature termination at $\mathrm{nt}$ 3651-3653, a one base insertion in exon 19 (5998insT) leading to a premature termination at nt 6022-6024, and a base substitution at the splice donor site in intron 21 $(6151+1 G>A)$ leading to a truncated protein by skipping exon 20 and adding nine amino acids after exon $19 .{ }^{10}$ The deletions were identified by FISH analysis on metaphase spreads prepared from peripheral lymphocytes or lymphoblastoid cell lines, using a probe for NSDI (RP1-118M12) and

\section{Key points}

- Although Sotos syndrome has been shown to be caused by haploinsufficiency of NSD1, clinical features have not been reported in patients with proven NSDI haploinsufficiency.

- We analysed the phenotypic findings in five patients with intragenic NSDI mutations predicted to form a truncated NSD 1 protein and in 21 patients with a fairly common $\sim 2.2 \mathrm{Mb}$ deletion involving the entire NSD 1 gene.

- Overgrowth and advanced maturation in infancy to early childhood, mental retardation, hypotonia, hyperreflexia, and characteristic minor anomalies were present in patients with mutations and deletions, whereas major anomalies in the central nervous system (agenesis or hypoplasia of the corpus callosum), cardiovascular system (patent ductus arteriosus and atrial septal defect), and urinary system (vesicoureteric reflux, hydronephrosis, and small kidney) were exclusively exhibited by patients with deletions.

- The results suggest that clinical features in Sotos syndrome are classified into two major categories, those primarily caused by NSD 1 haploinsufficiency and those primarily ascribed to some factors, such as the dosage effects of genes other than NSD1, involved in the deletion.

an additional five probes defining regions flanking NSD $1 .^{10}$ They consisted of a $\sim 2.2 \mathrm{Mb}$ deletion in a newly identified patient and the previously described 19 patients, and a somewhat smaller deletion in one previously reported patient. ${ }^{10}$

Long term data were available for statural growth and mental development in several patients of both groups. The peak height SD score was significantly different between $<6$ years of age and $>6$ years of age in patients with mutations (3.3 (0.5) $v 1.9(1.30), \mathrm{n}=5, \mathrm{p}=0.01)$ and in those with deletions $(2.7(0.9) \vee 0.9(1.2), \mathrm{n}=11, \mathrm{p}=0.001)$. Similarly, $\mathrm{IQ} / \mathrm{DQ}$, though the longitudinal data were scanty, appeared to increase in later age in both patients with mutations and deletions; in particular, two patients (a boy and a girl) with mutations, though apparently delayed until early childhood, showed developmental catch up and attended standard schools, with the improvement of IQ/DQ from 60 at 4 years of age to 85 at 17 years of age and from 70 at 5 years of age to 90 at 16 years of age, respectively. 
Clinical assessment was performed, through a questionnaire sent to physicians, for a variety of features reported in Sotos syndrome. Physical growth was evaluated by the Japanese reference data ${ }^{13}$ (Ministry of Health, Labour, and Welfare) and expressed as SD score, to allow for the integration of growth data of different sexes and different ages. Overgrowth was regarded as positive, if statural growth exceeded $+2 \mathrm{SD}$ of the mean. Bone age was assessed by the TW-2 method, standardised for Japanese, ${ }^{14}$ and was regarded as advanced if bone age in infancy to childhood ( $=6$ years of age) was above the 97th centile of age matched, normal children. Premature eruption of teeth was evaluated as positive if the first tooth appeared before 4 months of age. Mental development was assessed to be retarded when intelligence/ developmental quotient (IQ/DQ) was estimated to be less than 80. Muscle tone, tendon reflex, and minor anomalies were evaluated clinically. Brain anomaly was examined by magnetic resonance imaging (MRI) or by less sensitive computed tomography (CT) when MRI was not available. Cardiovascular anomaly was investigated by echocardiography. Urinary anomaly was studied by renal ultrasound, together with voiding cystourethrography (VCG) performed for patients with a history of urinary tract infection indicative of vesicoureteric reflux (VUR). Clinical episodes, such as neonatal asphyxia, neonatal hypoglycaemia (blood glucose $<40 \mathrm{mg} / \mathrm{dl}$ ), neonatal jaundice (serum total bilirubin above the age and weight matched Japanese reference data), recurrent febrile convulsions (=three times), and the occurrence of malignancy, were taken from the hospital records, as were parental heights and ages at the time of the patients' birth.

The variables are expressed as median and range or as mean (SD), after examining the normality and the variance by the $\chi^{2}$ test and the F test, respectively. The statistical significance of the median was analysed by the Mann-Whitney U test, that of the mean by Student's $t$ test or by the paired $t$ test, and that of the frequency by Fisher's exact probability test; $p=0.05$ was considered significant.

\section{RESULTS}

The results are summarised in table 1 . There was no significant difference in the sex ratio or in the age between patients with mutations and those with deletions. Unfortunately, not all features were evaluated in each patient, because of the retrospective study using the questionnaire. Overall, however, the patients with point mutations had overgrowth and advanced maturation (accelerated bone age and premature tooth eruption) in infancy to early childhood, mental retardation, hypotonia, hyperreflexia, and various characteristic minor anomalies (fig 1A, B). Overgrowth and mental retardation became less remarkable at a later age. In addition, one patient had large ventricles with brain atrophy as well as neonatal asphyxia and hypoglycaemia, and another patient had brain atrophy. The patients with deletions also had similar profiles of growth and maturation, performance disturbance, and minor anomalies (fig IC, D), although they tended to be smaller and more retarded than patients with mutations. Furthermore, brain, cardiovascular, and urinary anomalies were frequently present. Besides large ventricles and/or brain atrophy, agenesis or hypoplasia of the corpus callosum was delineated in seven of 17 patients examined by MRI, while it was undetected in two patients investigated by CT scan. Cardiovascular anomaly was identified in 12 of 21 patients by echocardiography. Renal anomaly was found in six of 13 patients by ultrasonography and VUR was delineated in all the five patients who received VCG. In addition, neonatal problems and recurrent convulsions were often observed, and neuroblastoma was identified in one patient with a $\sim 2.2 \mathrm{Mb}$ deletion. Between patients with mutations and those with deletions, statistical significance was found for the mean values of peak height SD score $=6$ years of age and IQ/DQ $>6$ years of age, and for the frequen- cies of cardiovascular anomaly, urinary anomaly, neonatal jaundice, and recurrent convulsions. In the parents, height was higher in the mutation group than in the deletion group, whereas age at the time of the patients' birth was similar in the two groups.

\section{DISCUSSION}

We analysed clinical features in five patients with intragenic NSDI mutations predicted to form truncated NSDI protein and in 21 patients with submicroscopic deletions involving the entire NSDI gene. Since a common $\sim 2.2 \mathrm{Mb}$ deletion accounts for most of the proven NSDI haploinsufficiency in Japanese patients, it is likely that low copy repeat sequences reside in the regions flanking the NSDI gene, and that an unequal crossover or intrachromosomal recombination is prone to occur between the repeat sequences. It has been reported, however, that intragenic mutations rather than submicroscopic deletions are the major cause of Sotos syndrome in non-Japanese patients. ${ }^{15}$ At present, it remains unknown why the prevalence of mutations and deletions reported to date is different between Japanese and non-Japanese patients.

The results help to define clinical features primarily caused by NSDI haploinsufficiency. Among features observed, overgrowth and advanced maturation, performance disturbance, and various minor anomalies, as well as slowing of statural growth and amelioration of mental development in later age, would primarily be ascribed to NSDI haploinsufficiency, because they were frequently exhibited by patients with mutations as well as those with deletions. In addition, NSDI haploinsufficiency may also be involved in the development of large ventricles/brain atrophy, neonatal asphyxia, and hypoglycaemia; although they were present in a limited number of patients in both groups, it is known that haploinsufficiency of human developmental genes are usually associated with a wide range of penetrance and expressivity, depending on other genetic and environmental factors. ${ }^{16}$ Of these features, apparent developmental "catch up" is noteworthy, because such a phenomenon has not been reported previously. ${ }^{17}{ }^{18}$ However, IQ/DQ may have been underlined in infancy to early childhood, because of hypotonia, relative large size, and poor coordination, so that this clinically important matter awaits further studies.

The results also serve to identify clinical features primarily ascribed to some factors other than NSD1 haploinsufficiency. Agenesis or hypoplasia of the corpus callosum, cardiovascular and urinary anomalies, neonatal jaundice, and recurrent convulsions would primarily be the result of loss of specific disease genes, other than NSD1, with variable penetrance and expressivity, because they were absent in patients with mutations and frequently exhibited by patients with deletions. In support of this, the deleted region is known to harbour at least 21 genes, ${ }^{10}$ although characterisation of these genes remains poor in terms of clinical effects. It should be pointed out, however, that major anomalies may be overlooked in several patients. For example, mild abnormality of the corpus callosum would be undetected on CT scan and might be unrecognised on MRI scan, and mild subclinical VUR may remain unidentified without performing VCG. In this context, Schaefer et $a l^{5}$ have reported that, of 40 non-Japanese Sotos syndrome patients examined by MRI $(n=36)$ or CT $(n=4)$, one patient had agenesis of the corpus callosum, 12 patients had diffuse hypoplastic corpus callosum, and 23 patients had mild or partial abnormality of the corpus callosum. In addition, the prevalence of cardiac and urinary anomalies in non-Japanese patients, though it is apparently lower than that of Japanese patients, is estimated to be roughly 10 and $20 \%$, respectively. ${ }^{1-46}$ Considering that the prevalence of NSDI mutation is relatively high in non-Japanese Sotos patients, ${ }^{15}$ and that NSD1 expression is positive in the brain and kidney and negative in the heart, ${ }^{11}$ it might be possible that NSDI 
Table 1 Phenotypic comparison between patients with NSD 1 point mutations and those with deletions involving NSD1

\begin{tabular}{|c|c|c|c|}
\hline & Mutation & Deletion & $p$ value \\
\hline Total number of patients & 5 & 21 & \\
\hline Sex ratio (male:female) & $2: 3$ & $15: 6$ & 0.21 \\
\hline Age range (years) & $9.8(6.5-17.1)$ & $7.5(3.0-21.4)$ & 0.35 \\
\hline \multicolumn{4}{|l|}{ Growth and maturation } \\
\hline Birth length (SDS) & $1.5(1.4)(n=3)$ & $0.7(1.0)(n=19)$ & 0.25 \\
\hline Birth weight (SDS) & $1.4(0.8)(n=5)$ & $0.6(1.0)(n=21)$ & 0.14 \\
\hline Birth OFC (SDS) & $2.1(0.8)(n=3)$ & $0.9(1.2)(n=20)$ & 0.10 \\
\hline Gestational age (week) & $40(38-41)(n=5)$ & $40(36-42)(n=18)$ & 0.36 \\
\hline Overgrowth ( $<6$ years) & $5 / 5$ & $18 / 21$ & 0.90 \\
\hline Peak height (SDS) & $3.3(0.5)(n=5)$ & $2.2(1.2)(n=21)$ & 0.007 \\
\hline Overgrowth (>6 years) & $2 / 5$ & $2 / 11$ & 0.37 \\
\hline Peak height (SDS) & $1.9(1.3)(n=5)$ & $0.9(1.2)(n=11)$ & 0.14 \\
\hline Advanced bone age ( $=6$ years) & $1 / 1$ & $10 / 14$ & 0.73 \\
\hline Premature eruption of teeth & $2 / 3$ & $15 / 17$ & 0.95 \\
\hline \multicolumn{4}{|l|}{ Performance } \\
\hline Mental retardation ( $=6$ years) & $3 / 3$ & $9 / 9$ & - \\
\hline$I Q / D Q$ & $60 \pm 10(n=3)$ & $45 \pm 20(n=9)$ & 0.26 \\
\hline Mental retardation ( $>6$ years) & $3 / 4$ & $5 / 5$ & 0.91 \\
\hline$I Q / D Q$ & $78 \pm 12(n=4)$ & $57 \pm 12(n=5)$ & 0.03 \\
\hline Hypotonia & $3 / 5$ & $7 / 21$ & 0.58 \\
\hline Hyperreflexia & $1 / 3$ & $6 / 18$ & 0.75 \\
\hline \multicolumn{4}{|l|}{ Minor anomaly } \\
\hline Craniofacial features & $5 / 5$ & $21 / 21$ & - \\
\hline Coarse appearance & $(5 / 5)$ & $(20 / 20)$ & $(-)$ \\
\hline Large head & $(5 / 5)$ & $(18 / 20)$ & $(0.63)$ \\
\hline Prominent forehead & $(5 / 5)$ & $(20 / 20)$ & $(-)$ \\
\hline Receding frontal hair line & $(3 / 4)$ & $(13 / 17)$ & (0.77) \\
\hline Hypertelorism & $(4 / 4)$ & $(18 / 21)$ & $(0.58)$ \\
\hline Downward slanting fissures & $(4 / 4)$ & $(17 / 21)$ & $(0.47)$ \\
\hline Large ears & $(4 / 5)$ & $(16 / 20)$ & $(0.75)$ \\
\hline High arched palate & $(3 / 3)$ & $(17 / 18)$ & $(0.86)$ \\
\hline Other features & $5 / 5$ & $20 / 21$ & 0.81 \\
\hline Scoliosis & $(1 / 5)$ & $(5 / 21)$ & $(0.68)$ \\
\hline Large hands and feet & $(5 / 5)$ & $(20 / 21)$ & $(0.81)$ \\
\hline \multicolumn{4}{|l|}{ Major anomaly } \\
\hline Brain anomaly & $2 / 5$ & $12 / 19$ & 0.33 \\
\hline Large ventricle/brain atrophy & $(2 / 5)$ & $(5 / 19)$ & $(0.46)$ \\
\hline Agenesis/hypoplasia of CC & $(0 / 5)$ & $(7 / 19)$ & $(0.15)$ \\
\hline Cardiovascular anomaly & $0 / 5$ & $12 / 21$ & 0.03 \\
\hline PDA & $(0 / 5)$ & $(4 / 21)$ & $(0.40)$ \\
\hline ASD & $(0 / 5)$ & $(2 / 21)$ & $(0.65)$ \\
\hline PDA and ASD & $(0 / 5)$ & $(6 / 21)$ & $(0.24)$ \\
\hline Urinary anomaly & $0 / 5$ & $7 / 13$ & 0.05 \\
\hline Hypoplastic kidney & $(0 / 5)$ & $(1 / 13)$ & $(0.72)$ \\
\hline Hydronephrosis & $(0 / 5)$ & $(1 / 13)$ & $(0.72)$ \\
\hline Hydronephrosis and VUR & $(0 / 0)$ & $(4 / 5)$ & $(0.23)$ \\
\hline VUR & $(0 / 0)$ & $(1 / 5)$ & $(0.72)$ \\
\hline \multicolumn{4}{|l|}{ Others } \\
\hline Neonatal asphyxia & $1 / 5$ & $3 / 21$ & 0.60 \\
\hline Neonatal hypoglycaemia & $1 / 5$ & $4 / 21$ & 0.69 \\
\hline Neonatal jaundice & $0 / 5$ & $11 / 21$ & 0.04 \\
\hline Recurrent afebrile convulsions & $0 / 5$ & $13 / 21$ & 0.02 \\
\hline Malignancy & $0 / 5$ & $1 / 21$ & 0.81 \\
\hline \multicolumn{4}{|c|}{$\begin{array}{l}\text { The denominators indicate the number of patients examined for the presence or absence of each feature, } \\
\text { and the numerators represent the number of patients assessed to be positive for that feature. Thus, the } \\
\text { differences between the denominators and numerators denote the numbers of patients evaluated to be } \\
\text { negative for that feature. }\end{array}$} \\
\hline
\end{tabular}

mutations cause major anomalies, especially mild abnormalities of the corpus callosum and urinary system.

In addition, several matters appear to be worth pointing out in the present study. First, the body size tended to be smaller in patients with deletions than in those with mutations. This would primarily be because of the variation in the genetic height potential as indicated by the significant difference in the parental height between the two groups, although growth disadvantage caused by perturbation of relevant genes in the deleted regions as well as variation in the environmental factors may also have contributed to the growth difference. Secondly, mental development tended to be more retarded in patients with deletions than in those with mutations. It is uncertain, however, whether this is primarily the result of perturbation of relevant genes in the deleted regions and/or variation in genetic and environmental background, because the parental IQ was not estimated. Thirdly, neuroblastoma was found in one of the 26 patients. This prevalence $(3.8 \%)$ is in close agreement with the previous estimation of the frequency of tumour development in Sotos syndrome (3.9\%). ${ }^{19}$ Since tumour formation is also often found in other overgrowth disorders such as Simpson-Golabi-Behemel syndrome (SGBS ${ }^{20}$ and Beckwith-Wiedemann syndrome (BWS), ${ }^{21}$ a genetic factor for somatic overgrowth such as GPC3 mutation in SGBS, ${ }^{22}$ IGF-2 overexpression in BWS, ${ }^{23}$ and NSDI haploinsufficiency in Sotos syndrome may raise the susceptibility to the development of tumours. However, it is possible that somatic mutation of the normal NSDI allele is responsible for tumour formation. Moreover, since the neuroblastoma was detected in a patient with a common deletion, it is also possible that a 


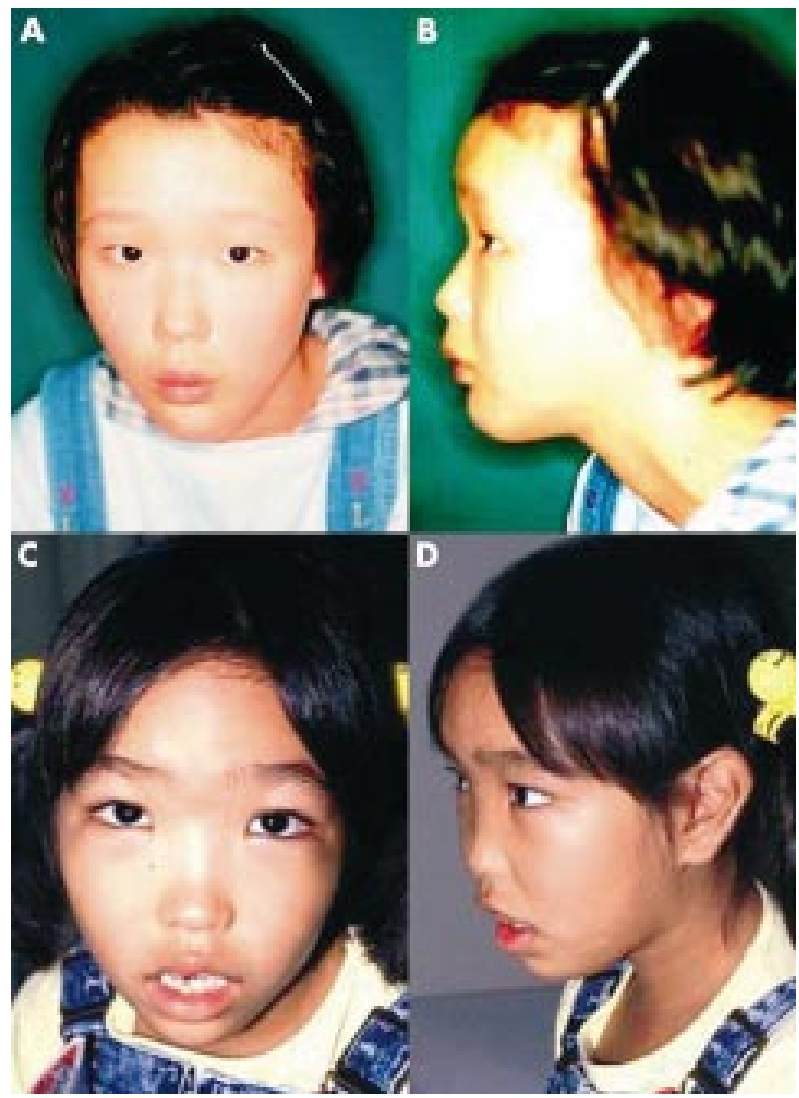

Figure 1 (A, B) A 9 year old girl with a NSD 1 point mutation. (C, D) An 8 year old girl with a deletion involving the entire NSD 1 gene.

tumorigenic gene(s) other than NSDI exists in the hemizygous region, and that haploinsufficiency of the gene(s) or nullizygosity of the gene(s) caused by somatic mutation is relevant to tumour formation. Molecular analysis of tumour tissues will provide a useful clue to this matter.

Several points should also be made with regard to the present study. First, the patients analysed, especially those with NSDl point mutations, are few in number. Thus, although significant $\mathrm{p}$ values were obtained for several variables, most of them were between 0.01 and 0.05 , so that further studies with a large number of patients would be necessary for a definitive conclusion. Secondly, since this study was based on a questionnaire, not all features were examined in each patient, as described in the Results section. Thirdly, since assessment of dysmorphic features is more or less subjective, some features may be underestimated or overestimated. Lastly, there may be a selection bias in that patients with fairly typical Sotos syndrome have been studied preferentially.

Despite the above caveats, the present study suggests that clinical features in Sotos syndrome may be classified into two major categories: those primarily caused by NSDI haploinsufficiency and those primarily ascribed to some other factors, such as the dosage effects of genes other than NSDI, involved in the deletion. Further genotype-phenotype correlations in both typical and atypical forms of Sotos syndrome will permit a better definition of the phenotypic spectrum in patients with NSD1 mutations, those with submicroscopic deletions involving NSDI, and those with normal NSDI.

\section{ACKNOWLEDGEMENTS}

This work was supported in part by a grant for Paediatric Research from the Ministry of Health and Labour, and a grant from CREST, Science, and Technology Corporation.

\section{Authors' affiliations}

T Nagai, Department of Paediatrics, Koshigaya Hospital, Dokkyo University School of Medicine, Koshigaya, Japan

N Matsumoto, N Kurotaki, N Harada, N Niikawa, Department of Human Genetics, Nagasaki University School of Medicine, Nagasaki Japan

T Ogata, Department of Endocrinology and Metabolism, National Research Institute for Child Health and Development, Tokyo, Japan K Imaizumi, K Kurosawa, Division of Medical Genetics, Kanagawa Children's Medical Centre, Yokohama, Japan

T Kondoh, Department of Paediatrics, Nagasaki University School of Medicine, Nagasaki, Japan

H Ohashi, Division of Medical Genetics, Saitama Children's Medical Centre, Iwatsuki, Japan

M Tsukahara, Faculty of Health Science, Yamaguchi University School of Medicine, Ube, Japan

Y Makita, Department of Paediatrics, Asahikawa Medical College,

Asahikawa, Japan

T Sugimoto, Department of Paediatrics, Kansai Medical University Otokoyama Hospital, Yawata, Japan

T Sonoda, Department of Paediatrics, Miyazaki Medical College, Miyazaki, Japan

T Yokoyama, Department of Paediatrics, Tokyo Metropolitan Kiyose Children's Hospital, Tokyo, Japan

K Uetake, Department of Paediatrics, Obihirokousei Hospital, Obihiro, Japan

S Sakazume, Y Fukushima, Department of Medical Genetics, Shinshu University School of Medicine, Matsumoto, Japan

K Naritomi, Department of Medical Genetics, University of Ryukyu School of Medicine, Okinawa, Japan

Correspondence to: Dr T Nagai, Department of Paediatrics, Koshigaya Hospital, Dokkyo University School of Medicine, 2-1-50

Minami-Koshigaya, Koshigaya-shi, Saitama 343-8555, Japan; t-nagai@dokkyomed.ac.jp

\section{REFERENCES}

1 Cole TRP, Hughes HE. Sotos syndrome. J Med Genet 1990;27:571-76.

2 Cole TR, Hughes HE. Sotos syndrome: a study of the diagnostic criteria and natural history. J Med Genet 1994;31:20-32.

3 Kaneko H, Tsukahara M, Tachibana H, Kurashige H, Kuwano A, Kajii T. Congenital heart defects in Sotos sequence. Am J Med Genet 1987;26:569-76.

4 Hammadeh MY, Dutta SN, Cornaby AJ, Morgan RJ. Congenital urological anomalies in Sotos syndrome. Br J Urol 1995;76:133-5.

5 Schaefer GB, Bodensteiner JB, Buehler BA, Lin A, Cole TRP. The neuroimaging findings in Sotos syndrome. Am J Med Genet 1997;68:462-5

6 Noreau DR, Al-Ata J, Jutras L, Teebi AS. Congenital heart defects in Sotos syndrome. Am J Med Genet 1998;79:327-8

7 Hersh JH, Cole TR, Bloom AS, Bertolone SJ, Hughes HE. Risk of malignancy in Sotos syndrome. J Pediatr 1992;120:572-4.

8 Cohen Jr MM. Tumors and nontumors in Sotos syndrome. Am J Med Genet 1999;84:173-5.

9 Winship IM. Sotos syndrome: autosomal dominant inheritance substantiated. Clin Genet 1995;28:243-6.

10 Kurotaki N, Imaizumi K, Harada N, Masuno M, Kondoh T, Nagai T, Ohashi H, Naritomi K, Tsukahara M, Makita Y, Sugimoto T, Sonoda T, Hasegawa T, Chinen Y, Tomita Ha HA, Kinoshita A, Mizuguchi T, Yoshiura Ki K, Ohta T, Kishino T, Fukushima Y, Niikawa N, Matsumoto N. Haploinsufficiency of NSDI causes Sotos syndrome. Nat Genet 2002;30:365-6.

11 Kurotaki N, Harada N, Yoshiura K, Sugano S, Niikawa N, Matsumoto N. Molecular characterization of NSD 1, a human homologue of the mouse Nsdl gene. Gene 2001;279:197-204

12 Huang N, vom Baur E, Garnier JM, Lerouge T, Vonesch JL, Lutz Y, Chambon $\mathrm{P}$, Losson $\mathrm{R}$. Two distinct nuclear receptor interaction domains in NSD1, a novel SET protein that exhibits characteristics of both corepressors and coactivators. EMBO J 1998;17:3398-412.

13 Suwa S, Tachibana K, Maesaka H, Tanaka T, Yokoya S. Longitudinal standards for height and height velocity for Japanese children from birth to maturity. Clin Pediatr Endocrinol 1992;1:5-13.

14 Murata M, Matsuo N, Tanaka T, Ohtsuki F, Ashizawa K, Tatara Y, Anzo M, Satoh M, Matsuoka H, Asami T, Tsukakoshi K. Radiographic atlas of skeletal development for the Japanese. Tokyo: Kanehara Press, 1993 (in Japanese).

15 Rahman N, Douglas J, Hanks S, Temple IK, Hughes HE, Cole T, and The Childhood Overgrowth Consortium. Intragenic mutations of NSDI are a major cause of Sotos syndrome and Weaver syndrome but do not account for other overgrowth phenotypes. Am J Hum Genet Supp 2002;71:169.

16 Fisher E, Scambler P. Human haploinsufficiency - one for sorrow, two for joy. Nat Genet 1994;7:5-7. 
17 Rutter SC, Cole TR. Psychological characteristics of Sotos syndrome. Dev Med Child Neurol 1991;33:898-902.

18 Finegan JK, Cole TR, Kingwell E, Smith ML, Smith M, Sitarenios G. Language and behavior in children with Sotos syndrome. J Am Acad Child Adolesc Psychiatry 1994;33:1307-15.

19 Gorlin RJ, Cohen MM, Levin LS. Overgrowth syndromes and postnatal onset obesity syndromes. In: Syndromes of the head and neck. 3rd ed. New York: Oxford University Press, 1990:323-52.
20 Neri G, Gurrieri F, Zanni G Lin A. Clinical and molecular aspects of the Simpson-Golabi-Behmel syndrome. Am J Med Genet 1998;79:279-83.

21 Wiedemann HR. Tumours and hemihypertrophy associated with Wiedemann-Beckwith syndrome. Eur J Pediatr 1983;141:129.

22 White GRM, Kelsey AM, Varley JM, Birch JM. Somatic glypican 3 (GPC3) mutations in Wilms' tumour. Br J Cancer 2002;86:1920-2.

23 Weksberg R, Shen DR, Fei YL, Song QL, Squire J. Disruption of insulin-like growth factor 2 imprinting in Beckwith-Wiedemann syndrome. Nat Genet 1993;5:143-50.

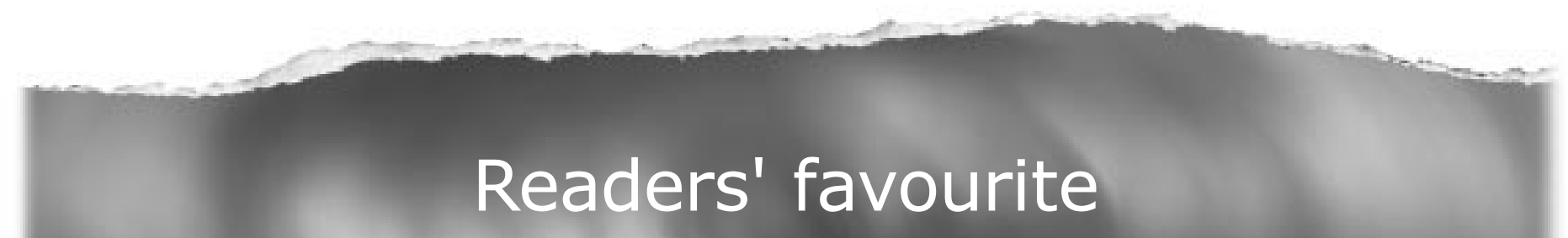

\section{Top 10}

Click on the "Top 10" button on the homepage to see which are the best read articles each month

\section{www.jmedgenet.com}

